\title{
Diffusion of a hydrocarbon mixture in a one-dimensional zeolite channel: an exclusion model approach
}

\author{
Sakuntala Chatterjee and Gunter M. Schütz \\ Institut für Festkörperforschung, Forschungzentrum Jülich, D-52425 Jülich, Germany
}

Abstract: Zeolite channels can be used as effective hydrocarbon traps. Earlier experiments (Czaplewski et al., 2002) show that the presence of large aromatic molecules (toluene) block the diffusion of light hydrocarbon molecules (propane) inside the narrow pore of a zeolite sample. As a result, the desorption temperature of propane is significantly higher in the binary mixture than in the single component case. In order to obtain further insight into these results, we use a simple lattice gas model of diffusion of hard-core particles to describe the diffusive transport of two species of molecules in a one-dimensional zeolite channel. Our dynamical Monte Carlo simulations show that taking into account an Arrhenius dependence of the single molecule diffusion coefficient on temperature, one can explain many significant features of the temperature programmed desorption profile observed in experiments. However, on a closer comparison of the experimental curve and our simulation data, we find that it is not possible to reproduce the higher propane current than toluene current near the desorption peak seen in experiment. We argue that this is caused by a violation of strict single-file behavior.

\section{INTRODUCTION}

Zeolites have wide industrial applications such as catalysts and adsorbents in many chemical and petro-chemical processes. It has been argued that a potential use for zeolites where adsorption and diffusion of molecules become important is as hydrocarbon traps for the automobile exhaust (see [1] and the references therein). When an automobile engine is turned on, the catalyst needs a certain time to reach its 'light-off' temperature, which is around $250-300^{\circ} \mathrm{C}$. This waiting time is known as cold-start period. During this period, a considerable amount of unburned hydrocarbon escape to the atmosphere through the tail-pipe. This loss of fuel is known as cold start problem. One possible solution to this problem is 


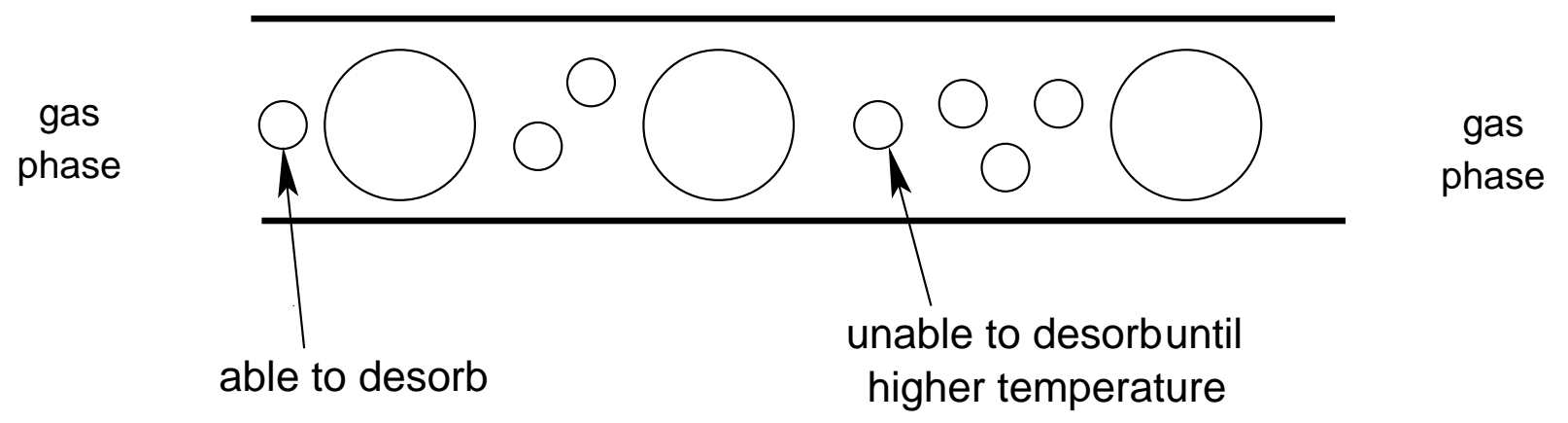

FIG. 1: Schematic diagram of 1-d zeolite pore to show the trapping of light, less-strongly adsorbed molecules by heavier, more-strongly adsorbed ones.

to use some trap to adsorb the hydrocarbon molecules and release them once the catalyst light-off has occurred. Zeolites are often used as effective hydrocarbon traps in this regard. However, it is often found that while the larger and heavier hydrocarbon molecules (e.g. aromatics) are trapped successfully inside the zeolite channels, the relatively light hydrocarbon components still manage to escape before the combustion temperature has been reached.

In [1] Czaplewski et al. have demonstrated that it is possible to administer the controlled release of light hydrocarbon molecules in the presence of large aromatic molecules using a one-dimensional zeolite channel. They have argued that in certain zeolite samples, the channels are so narrow that the hydrocarbon molecules cannot pass each other and the molecular transport takes place in essentially a single-file condition. In such effectively onedimensional zeolite channels, the more strongly adsorbed heavy aromatic molecules block the motion of the less strongly adsorbed light hydrocarbon molecules. Because of the confining pore dimension, the light hydrocarbon molecules cannot diffuse past the aromatic molecules (until higher temperature) and are thus trapped inside the zeolite pore, as shown in Fig 1. As a result, the light hydrocarbon molecules can desorb only after the aromatic molecules have desorbed which occurs at a higher temperature. This means that in the presence of large aromatic molecules the light hydrocarbon molecules desorb at a temperature which is much higher than the desorption temperature when no aromatic molecules are present.

Czaplewski et al. have performed an experiment using several zeolite samples with varying pore dimensionality and studied the desorption profile of propane (light hydrocarbon) and toluene (heavy aromatic molecule) mixture as the temperature is varied [1]. In conformity 
with the argument presented in the previous paragraph, they have found that inside a onedimensional zeolite channel, the desorption temperature of propane in a binary mixture is significantly higher than the single-component desorption temperature. However, for a zeolite with three dimensional connectivity, no such effect has been observed. This may be regarded as experimental verification that single-file diffusion is responsible for hydrocarbon trapping as described above.

It is the aim of this paper to provide a deeper theoretical understanding of the experimental observation of single-file diffusion, as reported in [1]. In particular, we are interested whether the assumption of single-file behavior alone is sufficient to explain the experimental data. Several different approaches have been used earlier to model the transport inside zeolites which include molecular dynamics simulations, dynamical Monte Carlo simulation, transition-state theory and Maxwell-Stefan approach [see [2] for a detailed review on this subject]. For the time-scales and length-scales that we are interested in, the method of dynamical Monte Carlo simulation is the most suitable approach. To this end we follow the strategy of modeling diffusion in zeolites as introduced by Kärger et al.[3, 4]. In the context of investigating so-called molecular traffic control [5] they used dynamical Monte Carlo simulation of a stochastic lattice gas model for zeolite systems with a network of perpendicular sets of intersecting channels (see [6-8] for recent progress along these lines). The basic idea of this model is to keep it as simple as possible with a minimum of adjustable parameters. In this paper, we use a similar lattice gas model, first introduced in $[9,10]$, to describe the single-file diffusion of a two-component mixture of molecules in a one-dimensional zeolite channel.

To give some background, we note that a binary mixture adsorbed inside a zeolite channel has been studied earlier using molecular dynamics simulation where the sorbate molecules are assumed to interact with one another and with the zeolite channel via a Lennard-Jones potential [11]. The self-diffusivities of the two species were compared against experimental values obtained from pulse field gradient NMR measurements. Both the simulations and experiments show that as the fraction of the strongly adsorbed species was increased (at constant total loading) the self-diffusivites of both components decrease (see also [12], where this concentration-dependence of single-file diffusivity is shown to affect the reaction kinetics inside a zeolite channel). In the simulation in [11], it was also found that the two different species show preferential adsorption at two different portions of the zeolite channel 
which tentatively supports the concept of molecular traffic control mentioned in the previous paragraph (see also [13]).

The attractive interaction between adsorbed molecules inside a zeolite pore is found to give rise to important collective effects even when the molecules of only a single species is present. In [14] the interaction between the neighboring adsorbate molecules was modeled by a Lennard-Jones potential and the effect of the sorbate-pore interaction was described by a sinusoidal potential. It was found that over a wide temperature range, the fragmentation of molecular clusters correspond to a higher energy barrier than the diffusion of a single molecule, indicating that molecular clusters are stable. More importantly, the energy barrier for cluster diffusion are often lower than that of a single-molecule diffusion. These results show that the single-file diffusion of molecules in a zeolite pore can be dominated by concerted movements of molecular clusters.

We have not attempted any detailed modeling of the interaction between sorbate molecules or between sorbate and zeolite. Instead we have worked with a simple lattice gas model and our Monte Carlo simulations show that within this simple model it is possi-

ble to explain major qualitative features of the experimental data of Czaplewski et al. [1]. From this we conclude that only details of the experimental curves are sensitive to details of the interactions among molecules and between molecules and the pore walls. However, it also transpires that the assumption of strict single-file diffusion fails to reproduce one important property of the experimental curves. This leads us to the further conclusion that some violation of the single-file condition occurs.

In the next section, we present a brief description of the experimental setting of Ref. [1] for the temperature programmed desorption (TPD) profile for propane-toluene binary mixture in one-dimensional zeolite samples with narrow (effectively, one-dimensional) pores. In section 3, we describe our model in detail, followed by our results and discussions in the section after.

\section{1-D ZEOLITES AS HYDROCARBON TRAPS: EXPERIMENT OF CZAPLEWSKI ET AL.}

Czaplewski et al. have considered several different zeolite samples with different pore sizes and various pore network connectivity. To study zeolites with one-dimensional channels, 
EUO and Na-Mor were used and these data were compared against the data obtained for Na-ZSM-5, which is a zeolite with three-dimensional connectivity. In this section, we briefly summarise the features of this series of experiments relevant for our study.

The zeolite samples were loaded with an equimolar binary mixture of propane and toluene and, for reference purposes, also with single-component propane and toluene separately. After the loading was complete, the whole system was purged in pure helium to remove all hydrocarbon molecules from the gas-phase portion of the flow system (see Fig 1). Then the sample was heated at a constant rate and the outflow was monitored using a flame ionisation detector and mass spectrometer.

For a single-component loading, it was found that as the temperature is steadily increased, the instantaneous output current rises, attains a peak and then falls off. The desorption temperature of each component was measured at the position of its desorption peak. For the one-dimensional zeolite Na-MOR, single-component toluene desorbs in two different stages (showing two different peaks) and this induces a two-stage desorption for the propane in the binary mixture. However, for the zeolite EUO, which also has a one-dimensional channel, desorption takes place in a single stage. For this simplicity, we focus on EUO and seek to describe its desorption profiles qualitatively using our model.

For the zeolite EUO, the single-component propane desorption peak is found at $40^{\circ} \mathrm{C}$ and for single-component toluene the peak occurs at $80^{\circ} \mathrm{C}$, toluene being more strongly adsorbed. For an equimolar binary mixture of the two gases in EUO, the propane desorption peak is found to occur at a substantially higher temperature $\left(75^{\circ} \mathrm{C}\right)$ and the toluene desorbs at $70^{\circ} \mathrm{C}$, as shown in Fig 2. Note that toluene desorption precedes the propane desorption, which suggests that in the narrow 1-d channel of EUO the propane molecules cannot pass the large toluene molecules and are able to desorb only after the toluene desorption peak has been reached.

This experiment demonstrates that using zeolite samples like EUO with 1-d channels, it is possible to trap light hydrocarbon molecules in presence of large aromatic molecules, until high temperature. No such effect has been observed for the 3-d zeolite Na-ZSM-5, which is consistent with the absence of single-file diffusion in this material. In the next section, we describe the lattice gas model that we use to explain the experimental data for EUO qualitatively. 


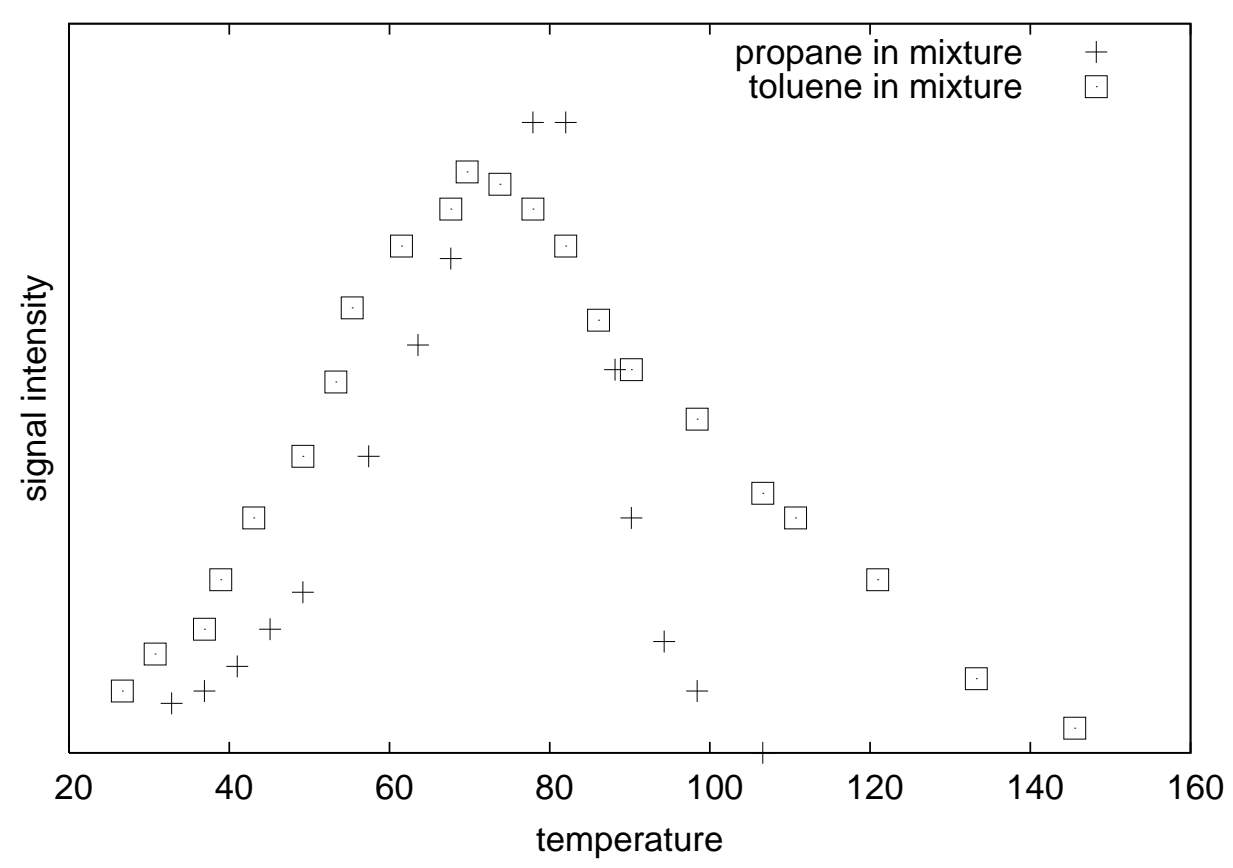

FIG. 2: Experimental data for TPD profile of propane and Toluene in binary mixture in zeolite EUO, as measured by Czaplewski et al. Data points taken from Fig 5 of [1].

\section{DESCRIPTION OF THE MODEL}

In the spirit of the approach by Kärger et al. [3, 4], we model the narrow pores of EUO by a one-dimensional lattice whose ends are open. In order to account for molecules of two different species with different diffusivities, we extend their approach and introduce two kinds of particles as follows. The diffusion of propane and toluene in the pore is modeled by a two-component symmetric exclusion process (SEP) on the lattice. We denote a propane molecule by the symbol ' $A$ ' and a toluene molecule by the symbol ' $B$ '. A site can either be occupied by an $A$ particle or a $B$ particle or can remain vacant (denoted by ' 0 '). An $A$ or $B$ particle can jump with rate $w_{A}$ or $w_{B}$, respectively, to the nearest neighbor site on either side, if the site is empty. If the neighboring site is occupied, no hopping is possible. The allowed moves are depicted in Fig 3. Note that in $[3,4]$, it was assumed that $w_{A}=w_{B}$ but we use different jump-rates (see Eq. 3) and this is one crucial aspect of our model. The dynamical moves in the bulk are: 


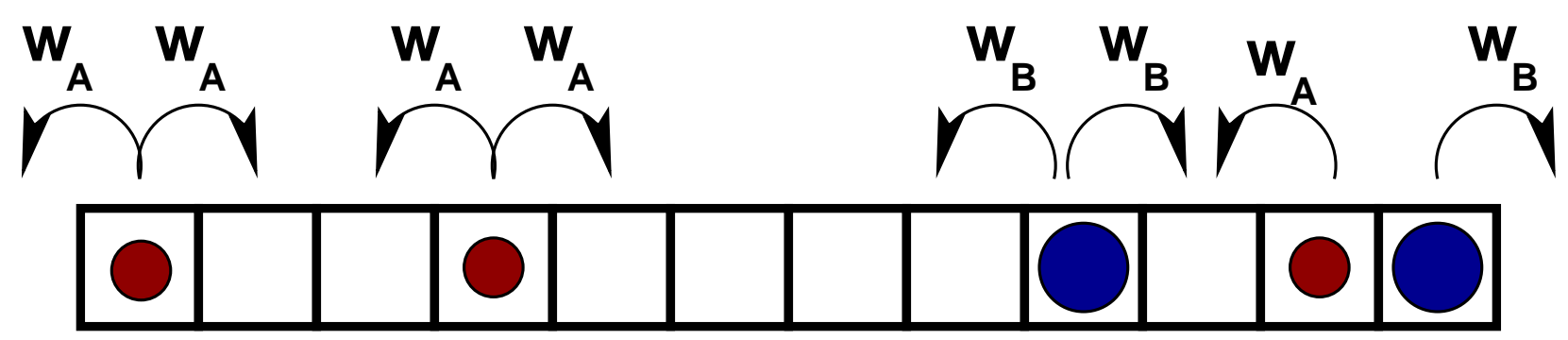

FIG. 3: Two component SEP on an open lattice

$$
\begin{aligned}
& A 0 \stackrel{w_{A}}{\longrightarrow} 0 A \\
& 0 A \stackrel{w_{A}}{\longrightarrow} A 0 \\
& B 0 \stackrel{w_{B}}{\longrightarrow} 0 B \\
& 0 B \stackrel{w_{B}}{\longrightarrow} B 0
\end{aligned}
$$

The $A$ or $B$ particles can exit through the boundary sites with rates $w_{A}$ and $w_{B}$, respectively. Therefore, the dynamical moves at the boundary are:

$$
\begin{aligned}
& A \stackrel{w_{A}}{\longrightarrow} 0 \\
& B \stackrel{w_{B}}{\longrightarrow} 0
\end{aligned}
$$

Once they exit, they are removed from the system and there is no boundary injection. Such a choice of boundary condition is motivated by the original experiment, where after the initial loading of the zeolite samples with hydrocarbon, in the course of the TPD measurement, hydrocarbons can desorb through the sample boundary, but no more hydrocarbons are loaded into the sample.

Eq. 1 defines a two-component SEP which describes the diffusion of two different species of hard-core particles. Note that when only a single particle $A(B)$ is present in an otherwise empty lattice, then the system shows normal diffusive behavior and the mean squared displacement (measured in units of lattice-spacing) is proportional to the elapsed time (measures in units of Monte Carlo step) with a proportionality constant $2 w_{A(B)}$. The twocomponent SEP was earlier studied in $[10,15]$ for non-zero rates for boundary injection and extraction, and constant values of $w_{A}$ and $w_{B}$. It was found that the equilibrium distribution of particles is uncorrelated, i.e. the probability that the $i$-th site contains an $A(B)$ 
particle $\operatorname{Prob}\left[n_{i}^{A(B)}=1\right]=\rho^{A(B)}$, independent of the occupancies of the other lattice sites. Here $\rho^{A(B)}$ refers to the reservoir density and the occupancy variable $n_{i}^{A(B)}$ is unity if $i$-th site is occupied by $A(B)$, and 0 otherwise.

In this paper, we consider time-dependent hopping rates $w_{A}$ and $w_{B}$ to model the TPD measurement carried out in experiment [1]. As time goes on, the temperature $T$ of the system is changed at a constant rate. The jump rates $w_{A}$ and $w_{B}$ are assumed to have the following Arrhenius dependence on temperature [18]:

$$
\begin{aligned}
& w_{A}=\Gamma_{A} \exp \left(-E_{A} / k T\right) \\
& w_{B}=\Gamma_{B} \exp \left(-E_{B} / k T\right)
\end{aligned}
$$

Here $k$ is the Boltzmann constant. Since Toluene is heavier, its diffusivity should be less than propane, i.e. $\Gamma_{A}>\Gamma_{B}$ and $E_{A}<E_{B}$. Note that in this two-component SEP, hopping rates are explicitly time-dependent which makes this problem difficult to deal analytically. In the case of constant (time-independent) rates, one can use a set of coupled non-linear differential equations to describe the time-evolution of the density profiles and can study stationary state properties and the relaxation towards steady state $[10,15]$. One might expect that this Maxwell-Stefan type approach can be extended to the time-dependent case as well, for very slow heating. However, this requires the presence of local equilibrium-an assumption we find difficult to justify in the present setting. Hence we use dynamical Monte Carlo simulation to study the system.

\section{SIMULATION RESULTS ON TWO COMPONENT SEP}

\section{A. Dynamical Monte Carlo Simulation}

In this section, we present our results of dynamical Monte Carlo simulations on the above lattice model. Assuming that the loading procedure generates an equilibrium state (homogeneous bulk density), we start from a random initial configuration drawn from the equilibrium ensemble, where each site can be occupied by an $A$ particles with probability $\rho_{A}$, by a $B$ particle with probability $\rho_{B}$, or remain vacant with probability $\left(1-\rho_{A}-\rho_{B}\right)$. The system is then evolved following the dynamical rules shown in Eqs. (1), (2). The 
'temperature' $T$ of the system is changed with time $t$ at a uniform rate:

$$
T=T_{0}+\lambda t
$$

where $T_{0}$ is the initial temperature and $\lambda$ is the increment in temperature per unit time. For each value of $T$, the jump rates are calculated from Eq. 3 .

One Monte Carlo time-step consists of $(L+1)$ update trials. At each update trial, we choose a lattice bond at random. If the bond lies in the bulk, the occupancies of the pair of sites adjacent to the bond are updated according to the rules given in Eq. 1: if one of these two sites contains an $A(B)$ and the other is empty, then a hopping across the bond takes place with probability $w_{A}\left(w_{B}\right)$. To perform this process, we draw a random number from a uniform distribution in the range $[0,1]$ and if the number is less than $w_{A}\left(w_{B}\right)$, then we exchange the occupancies of the pair of sites. If both the adjacent sites of the selected lattice bond are occupied, no action is taken. If the chosen lattice bond connects the boundary site (in this case, the leftmost or the rightmost site of the lattice) to the reservoir, then desorption takes place: if the boundary site is occupied by an $A(B)$ particle, then the site is emptied with a probability $w_{A}\left(w_{B}\right)$, following the same steps discussed above.

We measure the number of particles of each species coming out through the boundary sites per unit time, as a function of temperature (or time). We call this quantity instantaneous current and denote as $J_{A}(t)$ and $J_{B}(t)$, for outflow of $A$ and $B$, respectively.

\section{B. Single component loading}

When a single species, either $A$ or $B$ is present, then the system executes a singlecomponent SEP with time dependent rates. As temperature increases, the diffusivity increases and more and more particles come out from the lattice through the boundary sites. As a result the current increases. But since there is no boundary injection, the system starts getting depleted of particles and the current finally decreases after reaching a peak, as found in experiment [1].

Before we present our simulation results, a note about the choice of parameters is in order. Our aim is to provide a qualitative understanding of the experimental data. Within our simplistic model we do not expect an exact quantitative agreement with experiment. We have carried out simulation within the same temperature range as considered in the 
experiment and have chosen the activation energies and temperature increment rate $\lambda$ such as to obtain a desorption peak within this temperature range. However, the position of the desorption peak in our simulation does not match with the experimentally observed value.

In the experiment diffusion of propane and toluene was studied inside an EUO pore. Typical channel length of an EUO zeolite is $5 \mu \mathrm{m}$ and the molecular diameters of propane and toluene are, respectively, $\sim 4.4 \AA$ and $\sim 5.7 \AA$. The ratio of the channel length to the molecular size is $\sim 1000$, which should correspond to the number of lattice sites in our model. However, since our focus is to explain the main experimental results qualitatively, we work with a smaller lattice for computational efficiency. Also, the amount of substance adsorbed inside an EUO pore depends on the details of the structure of the pore and the adsorbed species. In our model, we have not taken into account of such details and have worked with an intermediate value of the density of $A$ and $B$ particles in the lattice. However, we have observed that even when a different value of the density is chosen, there is no qualitative change in our data (details not presented).

In our simulation, we have used a lattice of length $L=100$. In the initial configuration, each site contains an $A$ particle with a probability $\rho_{A}=0.4$. At the end of each Monte Carlo time-step, temperature is incremented by $5 \times 10^{-4}$ degree, starting from an initial value of $27^{\circ} \mathrm{C}$ to a final value $150^{\circ} \mathrm{C}$. We have used $E_{A}=83.1 \mathrm{~kJ} / \mathrm{mol}$. In order to make our simulation more efficient, the factor $\Gamma_{A}$ which sets the Monte Carlo time-scale, has been given a large value such that the variation of $w_{A}$ in the above temperature range is substantial. This is ensured by setting $\Gamma_{A}=\exp \left(E_{A} / k T_{f}\right)$, where $T_{f}$ is the final temperature. To obtain good statistics, we have averaged over 300,000 initial conditions. We present our data for $J_{A}(t)$ in Fig 4. In the same figure, the data for single-component loading of $B$ molecules is also shown, with $E_{B}=124.7 \mathrm{~kJ} / \mathrm{mol}, \rho_{B}=0.4$ and $\Gamma_{B}=\exp \left(E_{B} / k T_{f}\right)$. Note that the desorption peak for $J_{B}(t)$ occurs at a higher temperature than that for $J_{A}(t)$. In other words, $B$ is more strongly adsorbed than $A$, as expected.

\section{Loading of the binary mixture}

In the original experiment, zeolite samples were loaded with equimolar mixtures of propane and toluene. Likewise, we start with an initial configuration with $\rho_{A}=\rho_{B}$. Starting with a uniform mixture of $A$ and $B$, the plot of $J_{A}(t)$ and $J_{B}(t)$ is shown in Fig 5 against 


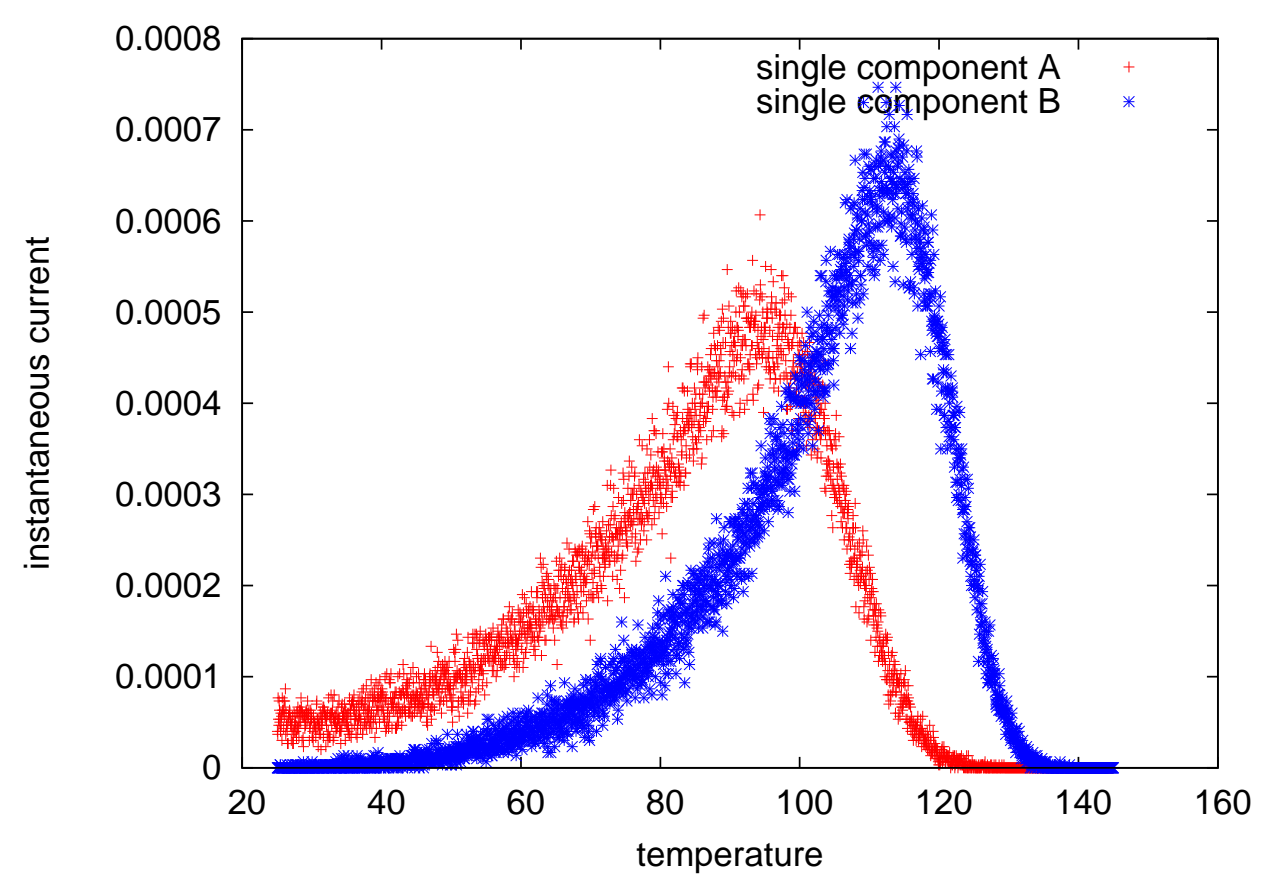

FIG. 4: Variation of instantaneous current as a function of temperature $\left(\right.$ in $\left.{ }^{\circ} C\right)$ for singlecomponent loading.

the variation of temperature.

We find that in the binary mixture, the positions of the peak of $J_{A}(t)$ and $J_{B}(t)$ almost coincide. The desorption temperature of $B$ is close to that in the single-component case, but desorption temperature of $A$ is substantially higher than the single-component case. This clearly shows that the presence of $B$ strongly influences the desorption of $A$. However, as seen from our data, the outflow of $A$ particles at low temperature is not suppressed much by the presence of $\mathrm{B}$. This means that a considerable fraction of $A$ particles still manages to escape at lower temperature. This happens due to the presence of $A$ molecules in the boundary region of the lattice which are not trapped by any $B$ molecule. When we start with a uniform mixture of $A$ and $B$ as the initial condition, there can be few $A$ molecules at the boundary, which do not have any $B$ molecules blocking their exit through the boundary.

We verify that if all such untrapped $A$ molecules are removed from the initial configuration, then the low temperature outflow of $A$ is suppressed (see Fig 6). We would like to comment that in the original experiment, after the zeolites were loaded with propane and toluene mixture, the system was passed through a 'gas-purging' phase [1]. In this phase, all the molecules were removed from the 'gas phase' (shown in Fig 1). It is very likely that the 


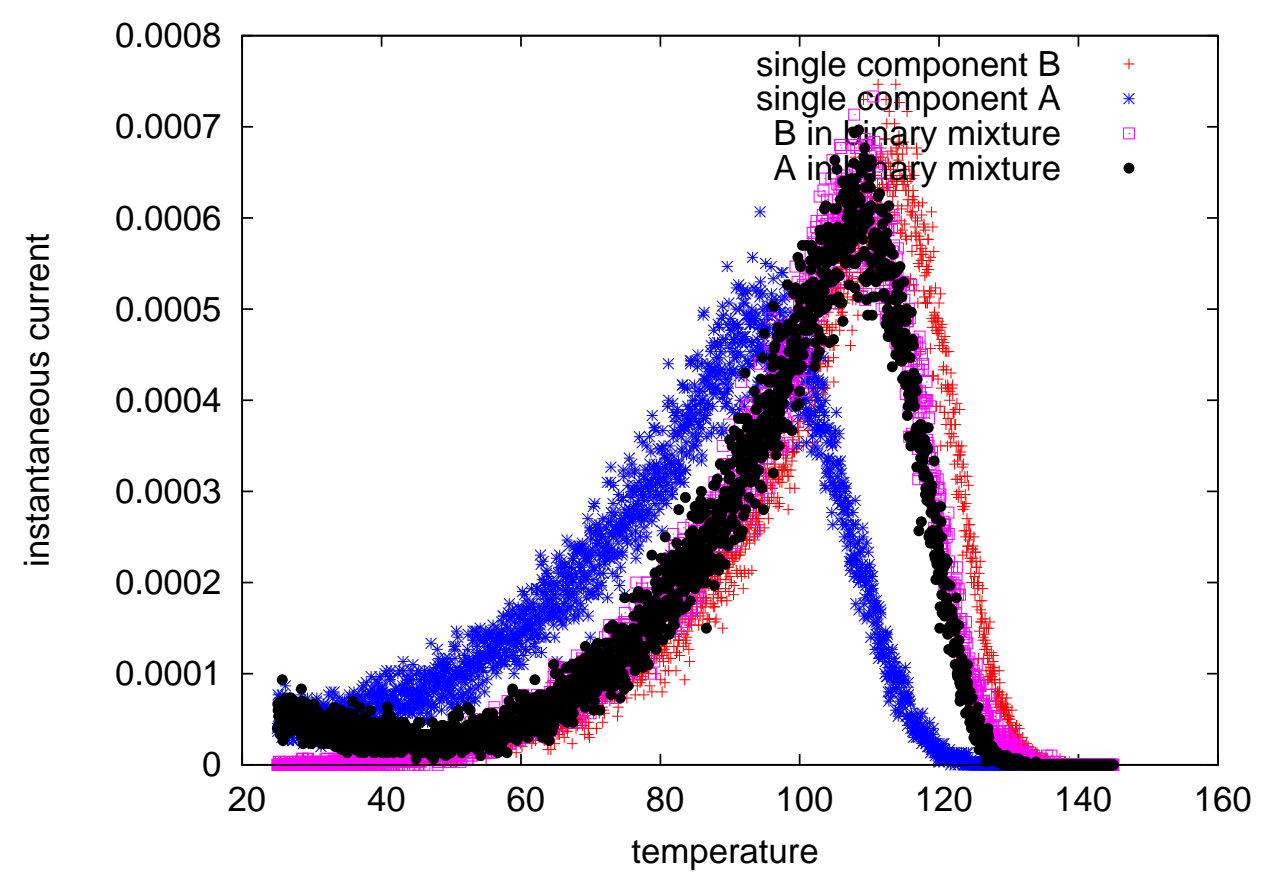

FIG. 5: Plot of current vs temperature $\left(\right.$ in ${ }^{\circ} C$ ) for a binary mixture of $A$ and $B$. We have used $L=100, \rho_{A}=\rho_{B}=0.4, \lambda=5 \times 10^{-4}{ }^{\circ} \mathrm{C}$ per unit time, $E_{A}=83.1 \mathrm{~kJ} / \mathrm{mol}, E_{B}=124.7 \mathrm{~kJ} / \mathrm{mol}$, $\Gamma_{A}=\exp \left(E_{A} / k T_{f}\right)$ and $\Gamma_{B}=\exp \left(E_{B} / k T_{f}\right)$. The data has been averaged over 300,000 initial configurations. For comparison, single-component data presented in Fig 4 has been replotted.

weakly adsorbed propane molecules which are untrapped by toluene molecules and reside close to the boundary sites, are also purged out of the system at this stage. So our choice of initial configuration with no untrapped $A$ particle, is not unrealistic but resembles the actual experimental scenario.

\section{Relaxing the single-file condition}

If we closely examine the experimental data shown in Fig 2, then we find that near the peak of the desorption profile the propane current is higher than the toluene current. The propane desorption peak is about $8 \%$ higher than that of toluene. However, in a strictly single-file condition, where propane molecules can escape only after the toluene molecules have desorbed, propane current can never exceed toluene current if we start from an equimolar mixture of the two [Fig 6].

Therefore, to explain the experimental observation, we have to modify our model of the 


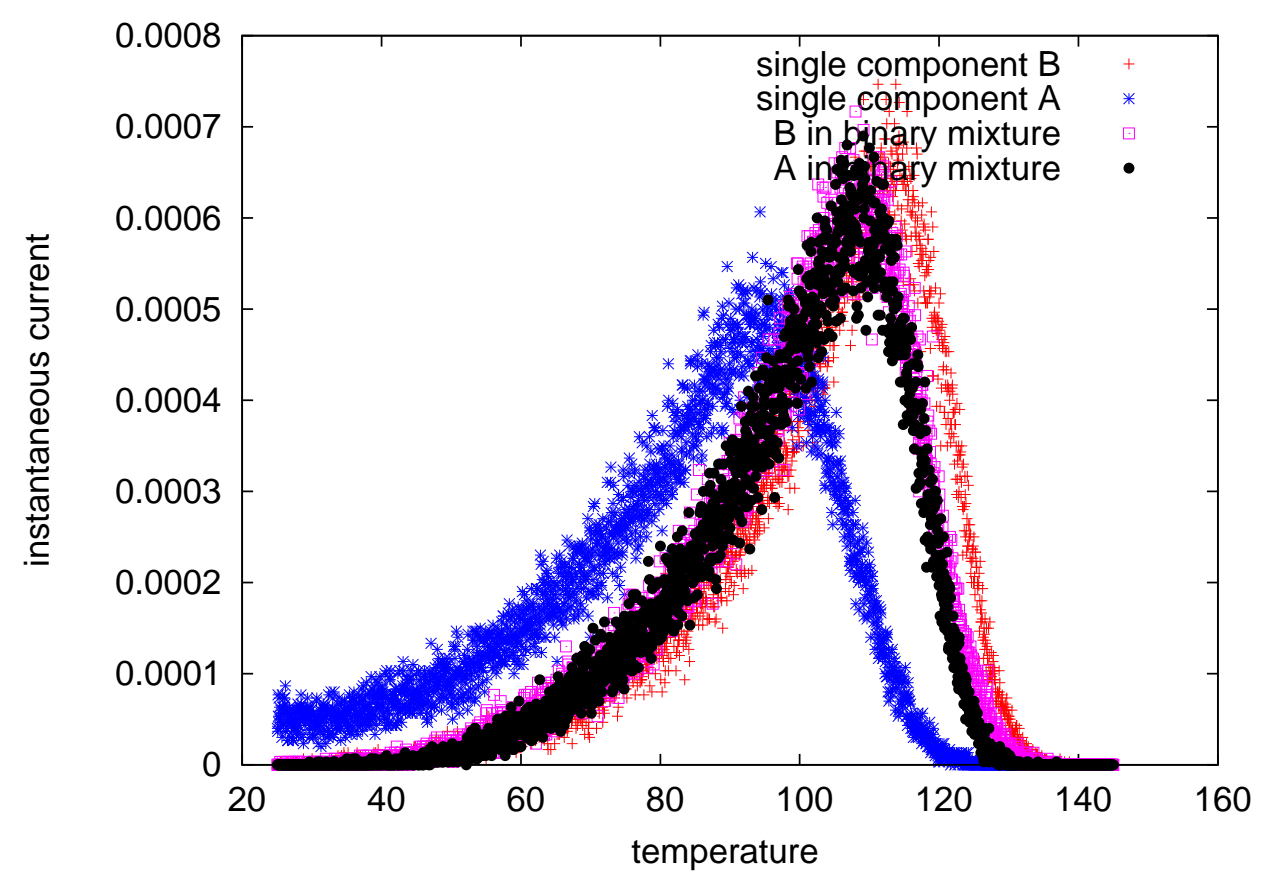

FIG. 6: The low temperature outflow of A particles, as shown in in Fig 5 is suppressed when all untrapped A particles are removed from the initial configuration. The other simulation parameters are same as in Fig 5.

exclusion process. To produce a higher propane current, as seen in experiment, we propose to slightly relax the single-file condition. In our model, we allow an $A$ particle to diffuse past a $B$ particle with a small rate, as shown in Fig 7 where

$$
w_{A B}=\Gamma_{A B} \exp \left(-E_{A B} / k T\right) .
$$

The other moves remain the same as in Eq. 1. Here, we choose $E_{A}<E_{B}<E_{A B}$ such that the violation of single-file condition becomes important only at sufficiently high temperature.

We present our simulation results with this modified model in Fig 8. We obtain a larger $A$ current than $B$ current near the peak, as observed experimentally. Our data shows that the desorption peak of $A$ is about $7 \%$ higher than that of $B$, close to the corresponding experimental value of $8 \%$. It is useful to have a rough estimate of the relative magnitudes of the different hopping probabilities $w_{A}, w_{B}$ and $w_{A B}$ in our simulation. With our choice of parameters, near the desorption peak, the ratio $w_{A B} / w_{B}$ attains a value of about 0.3 and $w_{A B} / w_{A}$ is close to 0.09 . We have found that the fraction of $A$ particles that desorb after crossing over a $B$ particle at the boundary, constitutes about $2.5 \%$ of the total current of $A$ 


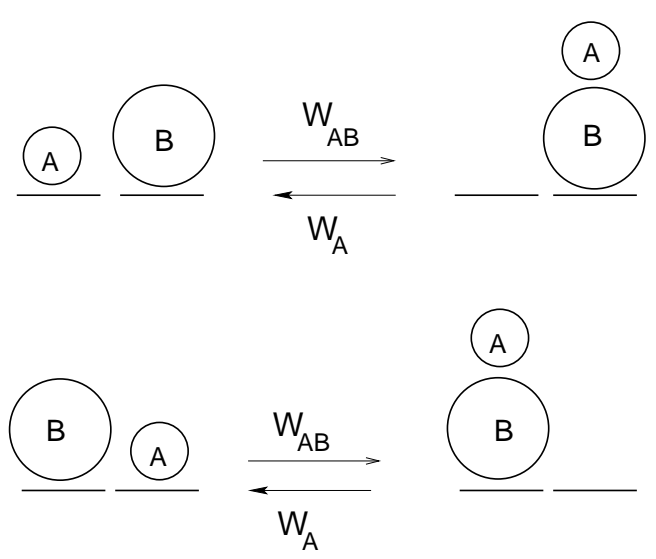

FIG. 7: A particles can cross over $B$ particles with a small rate when the single-file condition is relaxed.

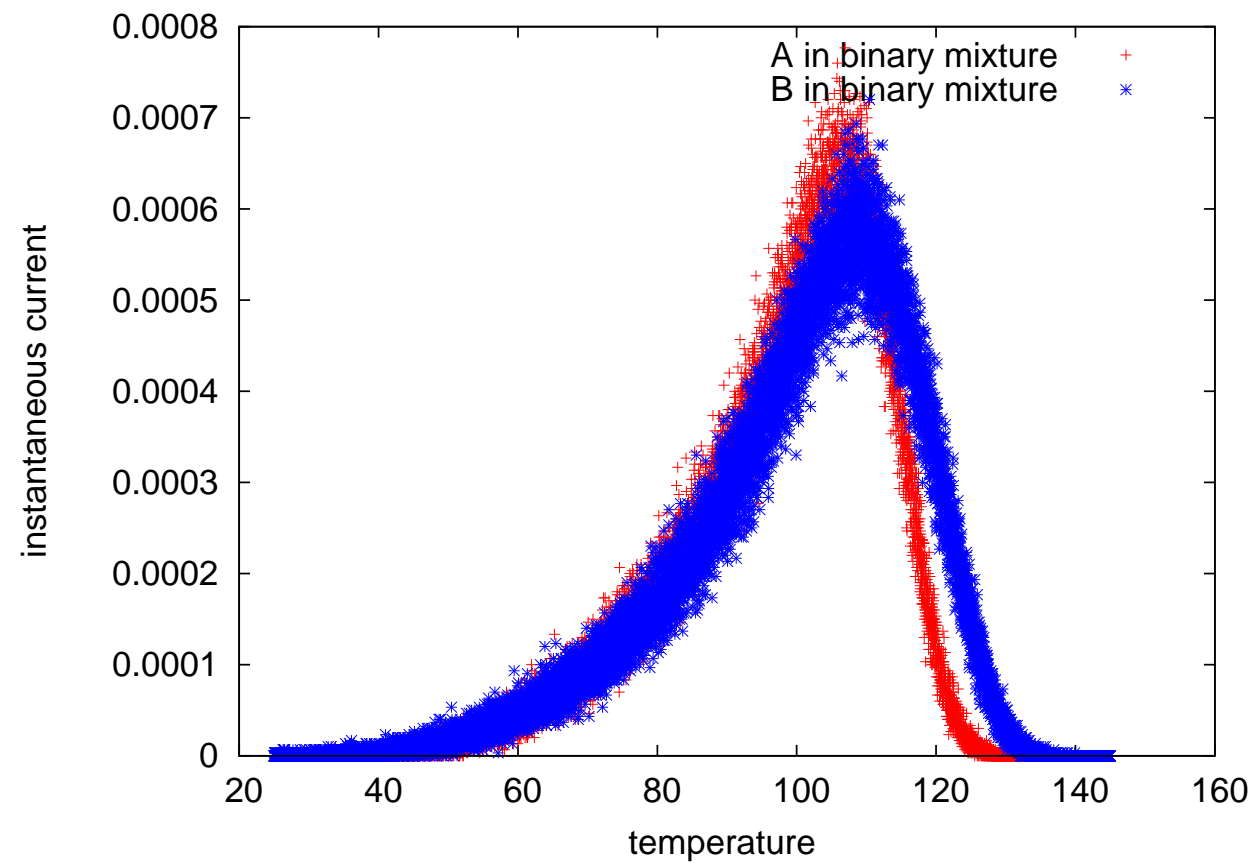

FIG. 8: Desorption profile for $A$ and $B$ with a relaxed single-file condition. We have used $E_{A B}=$ $166.2 \mathrm{~kJ} / \mathrm{mol}$ and $\Gamma_{A B}=\exp \left(E_{A B} / k T_{f}\right)$. The other simulation parameters remain same as in Fig 5.The data has been averaged over 300,000 'gas-purged' initial conditions.

particles, at the desorption peak. Hence the $A$ current has a rather small contribution from the crossing events at the boundary, but the desorption profile is nevertheless significantly affected by such crossings (see Fig 8). 


\section{CONCLUSION}

It is important to understand the transport mechanism of the molecules within the pores of zeolite in order to design more efficient applications for these materials. In this paper, we have used a two-component SEP to model the diffusion of propane and toluene in an effectively one-dimensional zeolite channel. Within this simple lattice gas model we have been able to explain the qualitative features of the temperature dependent desorption profile observed in experiment [1]. Our model for two-component single-file diffusion shows that the presence of strongly adsorbed $B$ particles raises the effective desorption temperature of weakly adsorbed $A$ particles, as seen in the original experiment. This indicates that no detailed modeling of the interaction between the molecules or between the molecule and the pore-wall and boundary effects are required to explain the main experimental results qualitatively.

Our Monte Carlo simulation shows that in order to reproduce a propane current larger than the toluene current, one has to relax the single-file condition and allow the particles to cross each other for sufficiently high temperature. A violation of the single-file condition in zeolite channels has been found earlier while studying tracer diffusion of methane molecules in $\mathrm{AlP}_{4}-5$ zeolite using molecular dynamics simulation [16]. The long-time growth of the mean squared displacement was found to be linear, as in normal diffusion. It was argued that because of narrow channels in $\mathrm{AlPO}_{4}-5$, the methane molecules are not able to cross each other easily, but infrequent crossings do take place. This gives rise to a diffusive regime on large time-scales. On short time-scales, an intermediate behavior was found where mean squared displacement is proportional to $t^{0.64}$. These results are in contrast with the experimental observation of single-file diffusion in $\mathrm{AlPO}_{4^{-}} 5$ [17]. In order to gain a better insight into these findings, a simplified "hop and cross" model was introduced that correctly reproduced the molecular dynamics results [16]. In this "hop and cross" model, a particle

jumps to the adjacent site with a probability $p_{\text {hop }}$ if the site is empty. If the neighboring site is occupied, then the two particles interchange their positions with a probability $p_{\text {cross }}$. In our model, where two different species are present, we have introduced a similar crossing effect by allowing the smaller particle to climb over the larger one (see Fig 7 ). We have found that near the desorption peak, the $A$ current has a rather small contribution from the crossing-events (about 2.5\%). However, such crossings do play a crucial role and affect the 
desorption profile, as seen in Fig 8. It would be interesting if it is possible to estimate the fraction of such crossing-events from the relative heights of the two peaks. However, we do not have much insight into this at present.

Throughout, we have assumed that the temperature dependence of the diffusivities $w_{A}$, $w_{B}$ and $w_{A B}$ follows the Arrhenius law (see Eqs. (1), (5)). This may not hold true. In fact for certain zeolites, non-Arrhenius temperature dependence has been discussed in earlier studies [18]. However, to our knowledge, such behavior for EUO zeolite has not been reported. Besides, our simple Arrhenius dependence yields good qualitative agreement with the experiment. Also, no qualitative difference is observed when the initial density is changed. Thus, within our model, we do not find any strong dependence on density. This conclusion is open to experimental verification.

In our model, we have not considered any interaction between the molecules, except hard-core exclusion. Since we can explain the main features of the experimental data qualitatively, it seems that the interaction details between the molecules do not play a crucial role. However, earlier studies have shown that these interactions can give rise to important collective effects [14] and hence might be relevant for a quantitative comparison with the experiment. In our model, we have also neglected any interaction between the molecules and the pore-wall. In particular, for an EUO zeolite, it is known that the narrow channel is accompanied by large side-pockets [19]. These side-pockets might give rise to a non-trivial interaction between the pore-wall and the molecules which in turn might affect the distribution for the residence-time of the molecules [20, 21]. It would be of interest to see what happens to the simulated desorption profile once the effect of the pore-wall interaction has been incorporated into our model.

\section{ACKNOWLEDGMENTS}

Financial support by the Deutsche Forschungsgemeinschaft within the priority programme SPP1155 is gratefully acknowledged. We would like to thank J. Kärger for inspiring discussions on single-file diffusion and diffusion in zeolites.

[1] K.F. Czaplewski et al., Micropor. Mesopor. Mater. 5655 (2002). 
[2] F.J. Keil, R. Krishna and M-O. Coppens, Rev. Chem. Eng. 1671 (2000).

[3] J. Kärger, P. Bräuer and H. Pfeifer, Z. Phys. Chem. 1041707 (2000).

[4] J. Kärger, P. Bräuer and A. Neugebauer, EuroPhys. Lett. 538 (2001).

[5] E.G. Derouane and Z. Gabelica, J. Catal. 65486 (1980).

[6] A. Brzank and G.M. Schütz, Appl. Catal. A: Gen. 288194 (2005).

[7] A. Brzank and G.M. Schütz, J. Chem. Phys. 124214701 (2006).

[8] R. Harish, D. Karevski, G.M. Schütz, J. Catal. 253191 (2008).

[9] T. Chou and D. Lohse, Phys. Rev. Lett. 823552 (1999).

[10] A. Brzank and G.M. Schütz, Diffus. Fundam. 4 7.1 (2006).

[11] R.Q. Snurr and J. Kärger, J. Phys. Chem. B 1016469 (1997).

[12] F.J. de Gauw, J. van Grondelle and R.A. van Santen, J. Catal. 20453 (2001).

[13] L.A. Clark, G.T. Ye and R.Q. Snurr, Phys. Rev. Lett. 842893 (2000).

[14] D.S. Sholl and K.A. Fichthorn, Phys. Rev. Lett. 793569 (1997).

[15] A. Brzank and G.M. Schütz, J. Stat. Mech. P08028 (2007).

[16] H.L. Tepper et al., J. Chem. Phys. 11011511 (1999).

[17] V. Kukla et al., Science 272702 (1996).

[18] A. Schüring et al., J. Chem. Phys. 11610890 (2002).

[19] W. Sorverijns et al., Micropor. Mesopor. Mater. 4123 (1995).

[20] S. Zschiegner et al., Diffus. Fundam. 717.1 (2007).

[21] K. Malek and M.-O. Coppens, J. Chem. Phys. 1192801 (2003). 